\title{
STOCHASTICALLY PERTURBED DYNAMICAL SYSTEMS
}

\section{WENDELL H. FLEMING ${ }^{1,2}$}

1. Introduction. A variety of natural phenomena have been modeled by stochastic differential equations containing one or more small parameters. Such models allow one to study the effects of random perturbations of small magnitude. When the parameters are set equal to 0 , the stochastic effects disappear from the problem; the resulting ordinary differential equations describe the evolution of the unperturbed dynamical system. Examples can be found in the study of wave propagation in random media [17], of noise in electronic systems [19] or in a control system [6]. Yet another class of examples, which we mention in $\$ 6$, arises from population genetics models.

For simplicity, we consider only one small positive parameter $\epsilon$. The state space of the system being modeled is taken as $n$-dimensional $R^{n}$. The state process $\xi^{\epsilon}=\left(\xi_{1} \epsilon, \cdots, \xi_{n}{ }^{\epsilon}\right)$ is assumed to be a Markov diffusion, with generator $\mathcal{L}^{\epsilon}$ the partial differential operator

$$
\mathcal{L}^{\epsilon} \psi=\epsilon \operatorname{tr} \alpha \psi_{x x}+\psi_{x} \cdot f
$$

Here $\psi$ denotes any function of class $C^{2}$ (continuous second order partial derivatives); the functions $\alpha, f$ are respectively matrix and vector valued:

$$
\begin{aligned}
\alpha(x) & =\left(\alpha_{i j}(x)\right), \quad i, j=1, \cdots, n, \\
f(x) & =\left(f_{1}(x), \cdots, f_{n}(x)\right) .
\end{aligned}
$$

The vector $\psi_{x}=\left(\psi_{x_{1}}, \cdots, \psi_{x_{n}}\right)$ is the gradient of $\psi$, and

$$
\operatorname{tr} \alpha \Psi_{x x}=\sum_{i, j=1}^{n} \alpha_{i j} \Psi_{x_{i} x_{j}}
$$

In fact, we shall take $\xi^{\epsilon}$ as the solution of a stochastic differential equation of Ito type $(\$ 2)$.

A variety of questions can be asked about the perturbed process $\xi \epsilon$. Which of these has a reasonable answer in a particular instance

${ }^{1}$ This research was supported in part by the Air Force Office of Scientific Research under Grant No. AF-AFOSR 71-2078, and in part by the National Science Foundation under Grant No. GP 15132.

${ }^{2}$ Presented at the Conference on Stochastic Differential Equations, University of Alberta, July 1972.

Received by the Editors June 22, 1973. 
often depends on the structure of the unperturbed system and on the way randomness enters. One question which one can always ask is the following: given a real valued function $\Phi$ on $R^{n}$, estimate the expected value $\boldsymbol{E} \Phi\left[\xi^{\epsilon}(t)\right]$ for fixed $t>0$ and small $\epsilon>0$. For instance, if $\boldsymbol{\Phi}(x)=|x|^{m}$, then $E\left|\xi^{\epsilon}(t)\right|^{m}$ is the $m$ th absolute moment.

In $\S 3$ a good approximation to $E \Phi\left[\xi^{\xi}(t)\right]$ is obtained in the form of an asymptotic expansion in powers of $\epsilon$. This result is a corollary of a theorem about expanding solutions of the Cauchy problem for the nonlinear parabolic equation $\left(4.1^{\epsilon}\right)$, whose second order coefficients contain the small positive parameter $\epsilon$.

If $\Phi$ is conserved in the unperturbed system, then it is interesting to estimate $E \Phi\left[\xi^{\epsilon}\left(t^{\epsilon}\right)\right]$, where $t^{\epsilon}=\mathrm{O}\left(\epsilon^{-1}\right)$ as $\epsilon \rightarrow 0$. Some rough estimates for the growth of this quantity are obtained in $\$ 3$ as an easy consequence of the Ito stochastic differential rule. Similar estimates were found previously by Carrier [1] using other methods. For systems of the type of a randomly perturbed harmonic oscillator occurring in wave propagation problems, a more exact description of the behavior of $\xi^{\epsilon}\left(t^{\epsilon}\right)$ is known [17].

In some problems, one is interested in the process $\xi \epsilon$ only up to the time $\tau^{\epsilon}$ when $\xi^{\epsilon}(t)$ exits from a given region $B$. One may then wish to estimate, for instance, $E \Phi\left[\xi^{\epsilon}\left(\tau^{\epsilon}\right)\right]$. This question is considered in $\$ 5$. We obtain an asymptotic expansion in powers of $\epsilon$. As is well-known in the theory of singular perturbations, such expansions cannot be expected to hold in all of $B$. Let us set $\varphi^{\epsilon}(x)=E_{x} \Phi\left[\xi^{\epsilon}\left(\tau^{\epsilon}\right)\right]$, where the subscript $x$ indicates the initial state $\xi(0)=x$. Then $\varphi$ satisfies for $\epsilon>0$ the elliptic second order partial differential equation $\mathcal{L}^{\epsilon} \varphi^{\epsilon}=0$ in $B$, with boundary data $\varphi^{\epsilon}=\Phi$ on $\partial B$. For $\epsilon=0$, the operator $\mathcal{L}^{0}$ is of first order. Some (or all) of the boundary data may be lost. The expansion in $\S 5$ is valid in portions of $B$ from which trajectories of the unperturbed dynamical system lead nontangentially to $\partial B$. It corresponds to the "regular expansion" obtained in the theory of singular perturbations by non-probabilistic methods [4]. It would seem interesting to study probabilistically boundary layer expansions, or other asymptotic properties of $\varphi^{\epsilon}$, outside regions where a regular expansion holds. However, we have not done so.

The equation $\mathcal{L}^{\epsilon} \varphi^{\epsilon}=0$ is linear. The probabilistic method to get regular expansions can also be used when $\varphi^{\epsilon}$ satisfies some nonlinear elliptic equation. When the nonlinearity involves only $\varphi^{\epsilon}$ (see (5.1є), or the corresponding parabolic equation $\left(4.1^{\epsilon}\right)$ ) the expansion problem is scarcely more difficult than for linear equations. The only additional step is to prove convergence of $\varphi^{\epsilon}$ to $\varphi^{0}$ as $\epsilon \rightarrow 0$ in regions where the regular expansion holds. If the nonlinearity involves the gradient $\varphi_{x}^{\epsilon}$, then one has to prove convergence of $\varphi_{x}^{\epsilon}$ to $\varphi_{x}^{0}$ as $\epsilon \rightarrow 0$. This is 
substantially more difficult. In certain cases it was done in [6], by writing the equation as the dynamic programming equation of a stochastic control problem.

2. The system equations. Let the Markov process $\xi^{\epsilon}$ satisfy the stochastic differential equations

$$
d \xi^{\epsilon}=f\left[\xi^{\epsilon}(t)\right] d t+(2 \epsilon)^{1 / 2} \boldsymbol{\sigma}\left[\xi^{\epsilon}(t)\right] d w, \quad t \geqq 0
$$

where $w$ is a brownian motion of some finite dimension. As initial data, let

$$
\xi(0)=x,
$$

where $x$ is a given vector. Occasionally, we write $\xi \epsilon(t ; x)$ to indicate dependence on the initial state $x$. More often, this dependence is shown by writing probabilities and expectations as $P_{x}(\cdots), E_{x}(\cdots)$. For $\epsilon=0$, we get the unperturbed system equations for the (nonrandom) function $\xi^{0}$.

$$
d \xi^{0}=f\left[\xi^{0}(t)\right] d t, \quad t \geqq 0,
$$

with the same initial data $\xi^{0}(0)=x$.

Let us assume that the functions $f, \sigma$ (respectively vector, matrix valued) are of class $C^{\infty}$. Moreover, we assume that there exist a positive constant $M$ and a positive $C^{\infty}$ function $V$ such that:

$$
\begin{gathered}
|\sigma(x)| \leqq M(1+|x|) \\
\mathcal{L}^{\epsilon} V \leqq M(1+V), \quad 0 \leqq \epsilon \leqq 1 \\
(1+|x|)\left|V_{x}\right| \leqq M(1+V) \\
V(x) \rightarrow \infty \text { as }|x| \rightarrow \infty .
\end{gathered}
$$

In the formula (1.1) for $\alpha$, take $\alpha=\sigma^{T}$. Then $f, 2 \epsilon \alpha$ are respectively the local drift and local covariance coefficients for the Markov process $\xi \epsilon$. These assumptions imply that $\xi^{\epsilon}$ is defined for all $t \geqq 0$. Moreover, for any $\ell=1,2, \cdots$, there is a bound on $E_{x} V^{\ell}\left[\xi^{\epsilon}(t)\right]$ depending only on $\ell$, a bound for $V(x)$, and $t$. See Appendix (A.2).

We shall use the following estimate, which shows that $\xi^{\epsilon}(t)$ is near $\xi^{0}(t)$ with probability very nearly 1 . A proof is given in the Appendix.

LeMma 2.1. Let $\left\|\xi^{\epsilon}-\xi^{0}\right\|_{t}=\max _{0 \leqq s \leqq t}\left|\xi^{\epsilon}(s)-\xi^{0}(s)\right|$. Then given $a>0$ a compact set $K \subset R^{n}$, and $t_{1}>0$, there exist $\lambda>0$, and $\epsilon_{1}>0$ such that

$$
P_{x}\left(\left\|\xi^{\epsilon}-\xi^{0}\right\|_{t}>a\right) \leqq 2 n \exp \left(-a \epsilon^{-1 / 2} \lambda\right)
$$

for all $x \in K, 0 \leqq t \leqq t_{1}$, and $0<\epsilon \leqq \epsilon_{1}$. 
We also need the following probabilistic representation for solutions $\psi(t, x)$ of a linear partial differential equation

$$
-\psi_{t}+\mathcal{L}^{\epsilon} \psi+A(t, x) \psi+g(t, x)=0 .
$$

Lemma 2.2. Let $G \subset R^{n}$ be an open set, and $\psi$ a solution of (2.5) in the cylinder $Q=(0, t) \times G$, such that $\psi$ is continuous and bounded in the closure $\bar{Q}$ while the partial derivatives $\psi_{t}, \psi_{x_{i} x_{j}}, i, j=1, \cdots, n$, are continuous in $Q$. Assume that $A, g$ are continuous and bounded on $Q$. Let $\tau$ denote the minimum of $t$ and the exit time of $\xi(s)$ from G. Then

$$
\begin{gathered}
\psi(t, x)=E_{x} \int_{0}^{\tau} D(s) g\left(t-s, \xi^{\epsilon}(s)\right) d s \\
+E_{x}\left[D(\tau) \psi\left(t-\tau, \xi^{\epsilon}(\tau)\right)\right] \\
D(s)=\exp \int_{0}^{s} A\left(t-u, \xi^{\epsilon}(u)\right) d u .
\end{gathered}
$$

This result follows rather easily from the Ito stochastic differential rule (Appendix). An important case is $A=0$; then $D(s)=1$. If, in addition, $g=0$, then $\psi$ is a solution of the backward equation $-\psi_{t}+$ $\mathcal{L}^{\epsilon} \psi=0$ for the diffusion process $\xi^{\epsilon}$. This special case is of interest in $\$ 3$.

When $g$ and $\psi$ are autonomous and $A \equiv 0$, Lemma 2.2 has the following corollary, which will be useful in $\S 5$.

LeMma 2.3. Let $G \subset R^{n}$ be an open set, and $\tau$ the exit time of $\xi \epsilon(s)$ from $G$ starting at $x \in G$. Assume that $E_{x} \tau<\infty$, and that $g$ is continuous and bounded on $G$. Let $\psi$ be a $C^{2}$ solution of

$$
\mathcal{L}^{\epsilon} \psi+g(x)=0 \text { in } G,
$$

such that $\psi$ is continuous and bounded in $\bar{G}$. Then

$$
\psi(x)=E_{x} \int_{0}^{\tau} g[\xi \epsilon(s)] d s+E_{x} \psi\left[\xi^{\epsilon}(\tau)\right] .
$$

To get Lemma 2.3, introduce the bounded stopping time $\tau_{t}=$ $\min (t, \tau)$, and use Lemma 2.2. Then let $t \rightarrow \infty$.

3. Estimates for $E_{x} \Phi\left[\xi^{\epsilon}(t)\right]$. Given $t$, we are interested in various statements which can be made about the random variables $\xi \epsilon(t)$ for small $\epsilon>0$. These could be answered in principle from knowing the distribution of $\xi \epsilon(t)$, which by Lemma 2.1 is mostly concentrated near 
the point $\xi^{0}(t)$. Instead of trying to find this distribution, we estimate integrals of various functions $\Phi$ with respect to it. Thus we are concerned with expectations of the form $E_{x} \Phi\left[\xi^{\epsilon}(t)\right]$. We begin with some estimates for this quantity which can be obtained by elementary arguments from the Ito stochastic differential rule. Later in the section we discuss expansions in powers of $\epsilon$.

We assume that $\Phi$ is $C^{\infty}$, and that for some positive constants $M, \ell$

$$
\begin{gathered}
|\Phi| \leqq M\left(1+V^{\ell}\right), \\
(1+|x|)\left|\Phi_{x}\right| \leqq M\left(1+V^{\ell}\right), \\
(1+|x|)^{2}\left|\Phi_{x x}\right| \leqq M\left(1+V^{\ell}\right) .
\end{gathered}
$$

These assumptions hold, for instance, if $\Phi$ is a polynomial and $V$ a positive polynomial.

By the Ito stochastic differential rule,

$$
d \Phi\left[\xi^{\epsilon}(s)\right]=\mathcal{L}^{\epsilon} \Phi d s+(2 \boldsymbol{\epsilon})^{1 / 2} \Phi_{x} \boldsymbol{\sigma} d w .
$$

By taking $E_{x} \int_{0}^{t}$ and recalling that $\xi^{\epsilon}(0)=x$, we get

$$
E_{x} \Phi\left[\xi^{\epsilon}(t)\right]=\Phi(x)+E_{x} \int_{0}^{t} \mathcal{L}^{\epsilon} \Phi\left[\xi^{\epsilon}(s)\right] d s .
$$

By (2.3), (3.1a, c) and Appendix (A.2) the expectations exist in (3.2). By $(3.1 b)$,

$$
\left|\Phi_{x} \sigma\right|^{2} \leqq M_{1}\left(1+V^{2 \ell}\right)
$$

for some $M_{1}$. Since $E_{x} V^{2 \ell}[\xi(s)]$ is bounded for $0 \leqq s \leqq t$ (by Appen$\operatorname{dix}\left(\right.$ A.2) with $2 \ell$ in place of $\ell$ there), $E_{x}\left|\Phi_{x} \sigma\right|^{2}$ is also bounded. This implies

$$
E_{x} \int_{0}^{t} \Phi_{x}\left[\xi^{\epsilon}(s)\right] \sigma\left[\xi^{\epsilon}(s)\right] d w=0
$$

as required in (3.2).

In differentiated form (3.2) becomes, upon interchanging expectation with time integration,

$$
\frac{d}{d t} E_{x} \Phi\left[\xi^{\epsilon}(t)\right]=E_{x} \mathcal{L}^{\epsilon} \Phi\left[\xi^{\epsilon}(t)\right] .
$$

The left side is the time derivative of the quantity we wish to compute. Unfortunately, the right side involves the expectation of $\mathcal{L}^{\epsilon} \Phi$ rather than $\Phi$. Nevertheless, useful information can often be obtained from $\left(3.2^{\prime}\right)$. 
Example 1. Consider a linear system

$$
d \xi=A \xi d t+(2 \epsilon)^{1 / 2} \sigma d w, \quad \sigma=\text { constant. }
$$

The process $\xi(=\xi \epsilon)$ is gaussian. The distribution of $\xi(t)$ is determined by the means and covariances

$$
\mu_{i}(t)=E_{x} \xi_{i}(t), v_{i j}(t)=E_{x}\left(\xi_{i}(t)-\mu_{i}(t)\right)\left(\xi_{j}(t)-\mu_{j}(t)\right) .
$$

These can be computed using (3.2'), taking first $\Phi(x)=x_{i}$, then $\Phi(x)=x_{i} x_{j}, i, j=1, \cdots, n$. One gets for the mean vector $\mu(t)=$ $\left(\mu_{1}(t), \cdots, \mu_{n}(t)\right)$ and covariance matrix $v(t)=\left(v_{i j}(t)\right)$ the differential equations

$$
\begin{aligned}
& \frac{d \mu}{d t}=A \mu, \quad \mu(0)=x, \\
& \frac{d v}{d t}=A v+v A^{T}+2 \epsilon \alpha, \quad v(0)=0 .
\end{aligned}
$$

Conservative systems. Let us next discuss the special case when $\Phi \geqq 0$ and $\Phi_{x} \cdot f=0$. Then $\Phi\left[\xi^{0}(t)\right]$ is constant on each trajectory of the unperturbed system $\left(2 \cdot 1^{0}\right)$. In many cases, $\Phi(x)$ can be regarded as the "energy" associated with the state $x$. Thus, we wish to estimate the average energy for the perturbed system

$$
e(t)=E_{x} \Phi[\xi \epsilon(t)] .
$$

By (1.1) we now have $\mathcal{L}^{\boldsymbol{\epsilon}} \boldsymbol{\Phi}=\boldsymbol{\epsilon} \operatorname{tr} \alpha \Phi_{x x}$. Since $\boldsymbol{\epsilon}$ appears as a coefficient on the right side of $\left(3.2^{\prime}\right)$, it is reasonable to anticipate that $e(t)$ will differ appreciably from $e(0)$ for times $t$ of order $\epsilon^{-1}$. An interesting question is the rate of growth of $e(t)$ as a function of $\epsilon t$. Some easy estimates of this type were discussed in [7], and applied to two examples of Carrier [1]. We shall now carry that discussion a bit further.

ExAMPLE 2. Let $n=2$,

$$
d \xi_{1}=\xi_{2} d t, d \xi_{2}=-\xi_{1} d t+(2 \epsilon)^{1 / 2} \xi_{1} d w .
$$

This is a randomly perturbed harmonic oscillator. Let $\Phi\left(x_{1}, x_{2}\right)=$ $(1 / 2)\left(x_{1}{ }^{2}+x_{2}{ }^{2}\right)$. Then

$$
\operatorname{tr} \boldsymbol{\alpha} \Phi_{x x}=x_{1}^{2} \Phi_{x_{2}, x_{2}}=x_{1}^{2} .
$$

Since the right side is no more than $2 \Phi\left(x_{1}, x_{2}\right)$, we have from $\left(3.2^{\prime}\right)$ 


$$
\begin{gathered}
\frac{d e}{d t} \leqq 2 \epsilon e, \\
e(t) \leqq e_{0} \exp (2 \epsilon t)
\end{gathered}
$$

where $e_{0}$ is the energy of the unperturbed system. This estimate is not sharp. A more careful analysis shows that the mean energy grows like $(1 / 2) \exp (\epsilon t)$. Consider the mean squared displacement $\mu(t)=$ $E\left[\xi_{1}(t)^{2}\right]$. Carrier [1] showed that $\mu(t) \sim(1 / 2) \exp (\epsilon t)$ as $\epsilon t \rightarrow \infty$. Since

$$
e(t)=e_{0}+\epsilon \int_{0}^{t} \mu(s) d s,
$$

we have also $e(t) \sim(1 / 2) \exp (\epsilon t)$ as $\epsilon t \rightarrow \infty$. The following (heuristic) argument also explains why the correct exponent is $\epsilon t$ rather than $2 \epsilon t$. When $\epsilon=0$

$$
\int_{t_{1}}^{t_{1}+2 \pi} \xi_{1}{ }^{0}(s)^{2} d s=\int_{t_{1}}^{t_{1}+2 \pi} \Phi\left[\xi^{0}(s)\right] d s,
$$

since the unperturbed system has as $2 \pi$-periodic orbits the circles $\Phi=$ constant. For small $\epsilon>0$ we should then have

$$
(* *) \quad \int_{t_{1}}^{t_{1}+2 \pi} \mu(s) d s \approx \int_{t_{1}}^{t_{1}+2 \pi} e(s) d s .
$$

One then replaces $\mu(s)$ by $e(s)$ in $(*)$, after writing the integral for $t=2 m \pi$ with $m$ large as the sum of integrals over periods. (The error in the approximation $(* *)$ could be estimated with the help of the expansion (3.5) for the mean energy and mean square displacement, conditioned on the state $\xi\left(t_{1}\right)$. However, we shall not carry out these estimates here.)

Considerably more is known about the oscillator problem in Example 2. By introducing as new variables the amplitude $r$ and phase shift $\phi$ and a new time scale $t^{\prime}=\epsilon t$, it can be shown that (in the new time scale) the $(r, \phi)$ process is closely approximated by a 2dimensional diffusion whose generator can be explicitly calculated. See [17]-[19] .

For our next class of examples we find an increasing function $u$, such that $u(e) \geqq 0$ for $e \geqq 0$ and

$$
E_{x} \operatorname{tr} \alpha \Phi_{x x} \leqq u[e(t)] .
$$

When such a function $u$ exists, from $\left(3.2^{\prime}\right) e$ satisfies the differential inequality 


$$
\frac{d e}{d t} \leqq \epsilon u(e) .
$$

If $F^{\prime}(e)=1 / u(e)$ and $e_{0}$ is the energy of the unperturbed system, we then have

$$
e(t) \leqq F^{-1}\left(F\left(e_{0}\right)+\epsilon t\right) .
$$

Example 3. Let us put a nonlinearity in Example 2, as follows:

$$
\begin{aligned}
d \xi_{1} & =\xi_{2} d t, d \xi_{2}=-\left(\xi_{1}+G^{\prime}\left(\xi_{1}\right)\right) d t+(2 \epsilon)^{1 / 2} \xi_{1} d w, \\
G\left(x_{1}\right) & =\Gamma\left(x_{1}{ }^{2}\right), \Gamma(0)=\Gamma^{\prime}(0)=0, \Gamma^{\prime \prime}>0 .
\end{aligned}
$$

Carrier considered in [1] the case $G^{\prime}\left(x_{1}\right)=x_{1}{ }^{3}, \Gamma(y)=(1 / 4) y^{2}$. We now take as energy function

$$
\Phi\left(x_{1}, x_{2}\right)=\frac{1}{2}\left(x_{1}{ }^{2}+x_{2}{ }^{2}\right)+G\left(x_{1}\right) .
$$

Again $\operatorname{tr} \alpha \Phi_{x x}=x_{1}{ }^{2}$. Take $u=\Gamma^{-1}$, the inverse of $\Gamma$. Then $u$ is a concave function, and $x_{1}^{2}=u\left[G\left(x_{1}\right)\right]$. Hence, writing $\xi_{1}(t)=\xi_{1}$,

$$
E_{x}\left(\xi_{1}\right)^{2}=E_{x} u\left[G\left(\xi_{1}\right)\right] \leqq u\left[E_{x} G\left(\xi_{1}\right)\right] .
$$

Since $u$ is increasing and $G \leqq \Phi$, we have

$$
E_{x} \operatorname{tr} \alpha \Phi_{x x}=E_{x}\left(\xi_{1}\right)^{2} \leqq u[e(t)],
$$

as required in (3.3). Thus the estimate (3.4) holds. In particular, if $G\left(x_{1}\right)=c x_{1}{ }^{2 m}, m \geqq 2$, then for suitable constant $c_{1}$

$$
\begin{aligned}
F^{-1}(r) & =c_{1} r^{m /(m-1)}, \\
e(t) & \leqq c_{1}(\epsilon t)^{m /(m-1)}
\end{aligned}
$$

for $\epsilon t$ large. The nonlinear term $-G^{\prime}\left(\xi_{1}\right) d t$ in the system equations imposes a much slower growth rate on the mean energy than in Example 2. It would be interesting to see whether this estimate might be improved, perhaps by using an averaging technique over periods of the unperturbed system. However, we have not succeeded in doing so.

Expansions in powers of $\epsilon$, for fixed $t$. We were concerned above with upper bounds for $E_{x} \Phi\left[\xi_{\epsilon}(t)\right]$, particularly for $t=t(\epsilon)$ of order $\epsilon^{-1}$. We now turn to the problem of making much sharper estimates for this quantity when $t$ is fixed. Let us write

$$
\varphi^{\epsilon}(t, x)=E_{x} \Phi\left[\xi^{\epsilon}(t)\right], \quad \varphi^{0}(t, x)=\Phi\left[\xi^{0}(t)\right] .
$$


We wish to expand $\varphi^{\epsilon}$ in powers of $\epsilon$ :

$$
\varphi^{\epsilon}=\varphi^{0}+\epsilon \theta_{1}+\epsilon^{2} \theta_{2}+\cdots+\epsilon^{k} \theta_{k}+o\left(\epsilon^{k}\right) .
$$

In $\S 4$ it will be shown that such an expansion is valid for any $k$ (finite). The coefficients $\theta_{1}, \theta_{2}, \cdots$ satisfy

$$
\theta_{k}(t, x)=\int_{0}^{t} \operatorname{tr} \alpha\left(\theta_{k-1}\right)_{x x} d s, \quad k=1,2, \cdots,
$$

where the integrand is evaluated at $\left(t-s, \xi^{0}(s)\right)$ and $\theta_{0}=\varphi^{0}$. One can derive formally equations (3.6) in the following way. Under perhaps more stringent growth conditions than (3.1) on $\Phi(x)$ for $|x|$ large, $\varphi^{\epsilon}$ must satisfy the partial differential equation

$$
-\varphi_{t}^{\epsilon}+\mathcal{L}^{\epsilon} \varphi^{\epsilon}=0, \quad t \geqq 0
$$

with $\varphi^{\epsilon}(0, x)=\Phi(x)$. By formally differentiating (3.7) repeatedly with respect to $\epsilon$ and setting $\epsilon=0$, one gets the first order partial differential equations

$$
-\left(\theta_{k}\right)_{t}+\mathcal{L}^{0} \theta_{k}+\operatorname{tr} \alpha\left(\theta_{k-1}\right)_{x x}=0
$$

with $\theta_{k}(0, x)=0$ for $k=1,2, \cdots$. The characteristic equations for (3.8) are just $\left(2.1^{\circ}\right)$, and thus we get formally (3.6).

To compute $\boldsymbol{\theta}_{1}, \boldsymbol{\theta}_{2}, \cdots$ for given initial state $x$, additional ordinary differential equations besides $\left(2.1^{\circ}\right)$ are needed. Let us explicitly indicate the dependence of $\xi^{0}$ on the initial state, by writing $\xi^{0}=$ $\xi^{0}(t, x)$. Then

$$
\varphi^{0}(t, x)=\Phi\left[\xi^{0}(t, x)\right] .
$$

To get $\theta_{1}$, one needs the matrix of second derivatives $\varphi_{x x}^{0}$. Partial derivatives $\varphi_{x_{i}}^{0}, \varphi_{x_{i} i_{i}}^{0}, \cdots$ of any order can be found from the derivatives of $\Phi$ and from $\xi_{x}^{0}, \xi_{x x}^{0}, \cdots$. The latter satisfy familiar ordinary differential equations obtained from $\left(2.1^{\circ}\right)$ by differentiation with respect to initial data. To get $\boldsymbol{\theta}_{2}$ one needs $\left(\boldsymbol{\theta}_{1}\right)_{x x}$. By differentiating (3.6) with $k=1$ with respect to $x_{i}$ and $x_{j}$, one gets $\left(\theta_{1}\right)_{x_{i} x_{j}}$ in terms of third and fourth order derivatives of $\varphi^{0}$; and so on. Unfortunately, the number of differential equations needed increases rapidly with $k$.

In the 1-dimensional case, there is a closed form expression provided $f\left[\xi^{0}(s)\right] \neq 0,0 \leqq s \leqq t$. In fact,

$$
\theta_{k}(t, x)=H_{k}\left[\xi^{0}(t, x), x\right],
$$


where

$$
\begin{gathered}
H_{0}(y, x)=\Phi(y), \\
H_{1}(y, x)=\frac{1}{[f(x)]^{2}} \int_{x}^{y} \alpha(z)\left\{\Phi_{y y}(z) f(z)+\Phi_{y}(z)\left[f^{\prime}(z)-f^{\prime}(x)\right]\right\} d z \\
H_{k+1}(y, x)=\frac{1}{f(x)} \int_{x}^{y} \alpha(z)\left\{H_{k y y} \frac{f(z)}{f(x)}\right. \\
\left.+2 H_{k y x}+H_{k x x} f(x)+H_{k y}\left[\frac{f^{\prime}(z)-f^{\prime}(x)}{f(x)}\right]\right\} d z .
\end{gathered}
$$

This can be shown by direct calculation.

4. Results for fixed $t$ (finite). Let us consider a second order partial differential equation of the form

$$
-\varphi_{t}^{\epsilon}+\mathcal{L}^{\epsilon} \varphi^{\epsilon}+F\left(x, \varphi^{\epsilon}\right)=0, \quad t \geqq 0,
$$

with the initial data

$$
\varphi^{\epsilon}(0, x)=\Phi(x) .
$$

When $F=0$, this becomes (3.7). For $\epsilon=0$, we have the first order equation

$$
-\varphi_{t}^{0}+\mathcal{L}^{0} \varphi^{0}+F\left(x, \varphi^{0}\right), \quad t \geqq 0,
$$

with the same initial data (4.2). The solution $\varphi^{0}$ can then be expressed by the method of characteristics.

Let us suppose that $\varphi^{\epsilon}$ is a bounded solution of $\left(4.1^{\epsilon}\right)-(4.2)$ with continuous partial derivatives $\varphi_{t}^{\epsilon}, \varphi_{x_{i}}^{\epsilon}, \varphi_{x_{i} x_{j}}^{\epsilon}, i, j=1, \cdots, n$. We wish to expand $\varphi^{\epsilon}$ in powers of $\epsilon$.

The coefficients in (3.5) must have the property that $k ! \theta_{k}$ is the $k$ th derivative of $\varphi^{\epsilon}$ with respect to $\epsilon$ when $\epsilon=0$. By formally differentiating $\left(4.1^{\epsilon}\right)$ repeatedly with respect to $\epsilon$, we get

$$
\begin{gathered}
-\left(\theta_{k}\right)_{t}+\mathcal{L}^{0} \theta_{k}+F_{\varphi} \theta_{k}+\Gamma_{k}^{0}+\operatorname{tr} \alpha\left(\theta_{k-1}\right)_{x x}=0, \\
\theta_{0}=\varphi^{0}, \Gamma_{1}^{0}=0, \quad \Gamma_{2}{ }^{0}=\frac{1}{2} \theta_{1}^{2} F_{\varphi \varphi}, \cdots .
\end{gathered}
$$

In general, $\Gamma_{k}{ }^{0}$ is a polynomial in $\theta_{1}, \cdots, \theta_{k-1}$ of degree $k$, with coefficients partial derivatives $F_{\varphi \varphi}, F_{\varphi \varphi \varphi}, \cdots$ evaluated at $\left(x, \varphi^{0}(x)\right)$. If $F=A(x) \varphi+g(x)$ is linear in $\varphi$, then all $\Gamma_{k}^{0}=0$.

THEOREM 4.1. Assume that $F_{\varphi}, F(x, 0)$, and $\Phi$ are bounded. Then the expansion (3.5) holds uniformly for $(t, x)$ in any compact set. 
Proof. Let

$$
\begin{aligned}
\theta_{1}{ }^{\epsilon} & =\epsilon^{-1}\left(\varphi^{\epsilon}-\varphi^{0}\right), \cdots, \theta_{k}^{\epsilon}=\epsilon^{-1}\left(\theta_{k-1}^{\epsilon}-\theta_{k-1}\right) \\
& =\epsilon^{-k}\left(\varphi^{\epsilon}-\varphi^{0}-\epsilon \theta_{1} \cdots-\epsilon^{k-1} \theta_{k-1}\right) .
\end{aligned}
$$

We must show that, for each $k, \theta_{k} \epsilon^{\epsilon}(t, x) \rightarrow \theta_{k}(t, x)$ as $\epsilon \rightarrow 0$, uniformly on any compact set. The functions $\boldsymbol{\theta}_{k}{ }^{\epsilon}$ satisfy linear partial differential equations (by direct calculation)

$$
\begin{gathered}
-\left(\theta_{k}^{\epsilon}\right)_{t}+\mathcal{L}^{\epsilon} \theta_{k}^{\epsilon}+A^{\epsilon} \theta_{k}^{\epsilon}+\Gamma_{k}^{\epsilon}+\operatorname{tr} \alpha\left(\theta_{k-1}\right)_{x x}=0 \\
A^{\epsilon}=\int_{0}^{1} F_{\varphi}\left(x, \varphi^{0}+\lambda\left(\varphi^{\epsilon}-\varphi^{0}\right)\right) d \lambda
\end{gathered}
$$

$$
\Gamma_{1}^{\epsilon}=0, \Gamma_{2}^{\epsilon}=\theta_{1} \theta_{1}^{\epsilon} \int_{0}^{1} \int_{0}^{1} F_{\varphi \varphi}\left(x, \varphi^{0}+\lambda \mu\left(\varphi^{\epsilon}-\varphi^{0}\right)\right) d \lambda d \mu, \cdots
$$

For $k>2, \Gamma_{k}{ }^{\epsilon}$ is a polynomial of degree $k$ in $\theta_{\ell}, \theta_{m}{ }^{\epsilon}, \ell, m \leqq k-1$, with coefficients involving $F_{\varphi \varphi}, F_{\varphi \varphi \varphi}, \cdots$ of orders up to $k$.

We first apply Lemma 2.2 to $\varphi^{\epsilon}$, with $A=0, g=F\left(x, \varphi^{\epsilon}\right), G=R^{n}$, to get

$$
\begin{aligned}
\varphi^{\epsilon}(t, x)= & E_{x} \int_{0}^{t} F\left[\xi^{\epsilon}(s), \varphi^{\epsilon}\left(t-s, \xi^{\epsilon}(s)\right)\right] d s \\
& +E_{x} \Phi\left[\xi^{\epsilon}(t)\right] .
\end{aligned}
$$

From the boundedness assumed for $F_{\varphi}$ and $F(x, 0), \quad\left|F\left(x, \varphi^{\epsilon}\right)\right| \leqq$ $C\left(1+\left|\varphi^{\epsilon}\right|\right)$ for some $C$. Using Gronwall's inequality we get from (4.5)

$$
\left\|\varphi^{\epsilon}(t, \cdot)\right\| \leqq(\|\Phi\|+C t) e^{C t},
$$

where \|\| is the sup norm. Therefore, $\varphi^{\epsilon}$ has a bound depending on $t$, but not $\epsilon$.

We next use Lemma 2.2 with $\psi=\theta_{1}{ }^{\epsilon}$ and $A=A^{\epsilon}, g=\operatorname{tr} \alpha \varphi_{x x}^{0}$, $G$ any open bounded set. We get

$$
\begin{aligned}
\theta_{1}^{\epsilon}(t, x)= & E_{x} \int_{0}^{\tau} D^{\epsilon}(s) \operatorname{tr} \alpha \varphi_{x x}^{0} d s \\
& +E_{x}\left[D^{\epsilon}(\tau) \theta_{1}^{\epsilon}\left(t-\tau, \xi^{\epsilon}(\tau)\right)\right], \\
D^{\epsilon}(s)= & \exp \int_{0}^{s} A^{\epsilon}\left(t-u, \xi^{\epsilon}(u)\right) d u,
\end{aligned}
$$

where $\operatorname{tr} \alpha \varphi_{x x}^{0}$ is evaluated at $(t-s, \xi \in(s))$. Given a compact set $K \subset R^{n}$ and $t_{1}>0$, choose $G$ large enough that $\xi^{0}(s) \in G$ for $0 \leqq s \leqq t_{1}$ 
if $\xi^{0}(0)=x$ and $x \in K$. Let $a$ be the minimum distance to $\partial G$ from any point $\xi^{0}(s), 0 \leqq s \leqq t$, with $x \in K$. By Lemma 2.1

$$
P_{x}(\tau<t) \leqq 2 n \exp \left(-a \epsilon^{-1 / 2} \lambda\right)
$$

for $0 \leqq t \leqq t_{1}$ and small $\epsilon$. Since $\varphi^{\epsilon}$ is bounded, $\left|\theta_{1}{ }^{\epsilon}\right| \leqq C_{1} \epsilon^{-1}$ for some $C_{1}$; and since $A^{\epsilon}$ is bounded (by boundedness of $F_{\varphi}$ ), $\left|D^{\epsilon} \theta_{1}^{\epsilon}\right| \leqq$ $C \epsilon^{-1}$ for some $C$. Moreover, $\theta_{1} \epsilon^{\epsilon}(t-\tau, \xi \epsilon(\tau))=0$ if $\tau=t$. It follows that

$$
\lim _{\epsilon \rightarrow 0} \mid E_{x}\left[D^{\epsilon}(\tau) \theta_{1}^{\epsilon}\left(t-\tau, \xi^{\epsilon}(\tau)\right) \mid \leqq C \lim _{\epsilon \rightarrow 0} \epsilon^{-1} P_{x}(\tau<t)=0 .\right.
$$

Thus, the second term on the right side of (4.6) tends to 0 . The first term there is bounded. Hence $\boldsymbol{\theta}_{1}{ }^{\epsilon}$ is bounded, which implies

$$
\lim _{\epsilon \rightarrow 0}\left(\varphi^{\epsilon}-\varphi^{0}\right)=\lim _{\epsilon \rightarrow 0} \epsilon \theta_{1}^{\epsilon}=0 .
$$

This holds uniformly on $\left[0, t_{1}\right] \times K$. By Lemma $2.1,\left\|\xi^{\epsilon}-\xi^{0}\right\|_{t}$ tends to 0 in probability as $\epsilon \rightarrow 0$. Moreover, $\tau$ tends to $t$ in probability. Therefore,

$$
\begin{gathered}
\lim _{\epsilon \rightarrow 0} E_{x} \int_{0}^{\tau} D^{\epsilon}(s) \operatorname{tr} \alpha \varphi_{x x}^{0} d s=\int_{0}^{t} D^{0}(s) \operatorname{tr} \alpha \varphi_{x x}^{0} d s, \\
D^{0}(s)=\exp \int_{0}^{s} A^{0}\left(s-u, \xi^{0}(u)\right) d u,
\end{gathered}
$$

where $\operatorname{tr} \alpha \varphi_{x x}^{0}$ on the right side is evaluated at $\left(t-s, \xi^{0}(s)\right)$. From the method of characteristics the right side is then the desired solution of $\left(4.3^{\circ}\right)$ when $k=1$. Thus, by (4.6), $\theta_{1}{ }^{\epsilon} \rightarrow \theta_{1}$ as $\epsilon \rightarrow 0$. The convergence is uniform on $\left[0, t_{1}\right] \times K$. This proves Theorem 4.1 for $k=1$.

The proof now continues by induction on $k$. Again by Lemma 2.2, we have

$$
\begin{aligned}
\theta_{k}^{\epsilon}(t, x)= & E_{x} \int_{0}^{\tau} D^{\epsilon}(s)\left[\Gamma_{k} \epsilon+\operatorname{tr} \alpha\left(\theta_{k-1}\right)_{x x}\right] d s \\
& +E_{x}\left[D^{\epsilon}(\tau) \theta_{k}^{\epsilon}\left(t-\tau, \xi^{\epsilon}(\tau)\right)\right] .
\end{aligned}
$$

Since $\varphi^{\epsilon}$ is bounded, $\left|\theta_{k}{ }^{\epsilon}\right| \leqq C_{k} \epsilon^{-k}$ for some $C_{k}$. This, together with (4.7), suffices to show that the extreme right hand side of (4.8) tends to 0 as $\epsilon \rightarrow 0$. Using the induction hypothesis, it can be shown that $\Gamma_{k} \epsilon^{\epsilon}$ tends to $\Gamma_{k}{ }^{0}$ uniformly on compact sets. It then follows as before that $\theta_{k}{ }^{\epsilon} \rightarrow \theta_{k}$ uniformly on $\left[0, t_{1}\right] \times K$ as $\epsilon \rightarrow 0$. This proves Theorem 4.1.

REMARK. In Theorem 4.1 we imposed conditions which insure that 
$\varphi^{\epsilon}$ is uniformly bounded for bounded $t$. The same method would give the expansion (3.5) under any weaker set of assumptions which guarantee that the solutions $\varphi^{\epsilon}$ of (4.1€)-(4.2) are uniformly bounded on compact sets.

Example. Consider the equation in 1 space variable

$$
-\varphi_{t}^{\epsilon}+\epsilon \varphi_{x x}^{\epsilon}+F\left(\varphi^{\epsilon}\right)=0 .
$$

Then $\alpha(x)=1, f(x)=0$. For $\epsilon=0, \varphi^{0}$ is a solution of the ordinary differential equation $\varphi_{t}^{0}=F\left(\varphi^{0}\right)$, depending on $x$ as a parameter. The function $\boldsymbol{\theta}_{1}$ is a solution of the linear ordinary differential equation

$$
\left(\theta_{1}\right)_{t}=F^{\prime}\left(\varphi^{0}\right) \theta_{1}+\varphi_{x x}^{0}
$$

One interpretation of equation (4.9) is as a simplified model of population growth with small diffusion. The population is distributed along an infinite linear habitat, and $\varphi^{\epsilon}(t, x)$ represents the population density at place $x$ and time $t$. Typical choices for $F$ are $F(\varphi)=m \varphi$ (linear growth rate) and $F(\varphi)=m_{1} \varphi-m_{2} \varphi^{2}$ (logistic growth rate). Another equation of type (4.9) arising from a population genetics model with diffusion was considered in [12]. That model neglects the effects of random sampling of genes in a population of finite size.

Expansion of $E_{x} \Phi\left[\xi^{\epsilon}(t)\right]$. We now apply Theorem 4.1 to obtain the expansion (3.5) already announced in \$3. There is a slight technical difficulty because we do not know, under the rather mild growth assumptions (3.1), that the function $\varphi^{\epsilon}$ defined in $\$ 3$ is actually a solution of the partial differential equation $\left(4.1^{\epsilon}\right)$.

THEOREM 4.2. If $\Phi$ satisfies assumptions (3.1), then the expansion (3.5) holds for $\varphi^{\epsilon}(t, x)=E_{x} \Phi\left[\xi^{\epsilon}(t)\right]$ uniformly for $(t, x)$ in any compact set.

Proof. Step 1. Suppose for the moment that $f, \sigma, \Phi$ are bounded together with their first and second order partial derivatives. Results about smooth dependence of solutions of stochastic differential equations on the initial state $x$ then imply that $\varphi^{\epsilon}$ has continuous partial derivatives $\varphi_{t}^{\epsilon}, \varphi_{x_{i}}^{\epsilon}, \varphi_{x_{i} x_{j}}^{\epsilon}$ and satisfies

$$
-\varphi_{t}^{\epsilon}+\mathcal{L}^{\epsilon} \varphi^{\epsilon}=0 .
$$

See [9, Chapter VIII.5] or [10, Part I, $\$ 11$, Theorem 1]. Moreover, $\left\|\varphi^{\epsilon}\right\| \leqq\|\Phi\|$. By Theorem 4.1 with $F=0$, expansion (3.5) holds uniformly on compact sets. 
Step 2. Now let $\Phi$ be as in Step 1, while $f$, $\sigma$ satisfy (2.3)-(2.4). Choose $f_{m}, \boldsymbol{\sigma}_{m}$ satisfying the conditions in Step 1 for $m=1,2, \cdots$, such that $f_{m}=f, \boldsymbol{\sigma}_{m}=\boldsymbol{\sigma}$ for $x \in G_{m}$, where $G_{m}$ is an expanding sequence of open sets with union $R^{n}$. Let

$$
\varphi_{m}{ }^{\epsilon}(t, x)=E_{x} \Phi\left[\xi_{m}{ }^{\epsilon}(t)\right],
$$

where $\xi_{m} \epsilon$ satisfies $\left(2.1^{\epsilon}\right)$ with $f_{m}, \sigma_{m}$ replacing $f, \boldsymbol{\sigma}$. These solutions can be so defined that $\xi_{m}{ }^{\epsilon}(t)=\xi^{\epsilon}(t)$ for $0 \leqq t \leqq \tau_{m}{ }^{\epsilon}$, where the exit time $\tau_{m}{ }^{\epsilon}$ from $G_{m}$ tends to infinity as $m \rightarrow \infty$. The following stronger estimate holds, in fact. Given a compact set $K$ and $t_{1}$, fix $G_{\mu}=G$ with $\mu$ large enough that the situation in the proof of Theorem 4.1 holds. Let $\tau$ be as in Lemma 2.2. Then

$$
\varphi_{m}{ }^{\epsilon}(t, x)=E_{x} \varphi_{m}{ }^{\epsilon}\left(t-\tau, \xi^{\epsilon}(\tau)\right), \quad m \geqq \mu,
$$

for $x \in G_{\mu}$. Moreover, $\left\|\varphi_{m}{ }^{\epsilon}\right\| \leqq\|\Phi\|$. Let $m \rightarrow \infty$; by dominated convergence

$$
\varphi^{\epsilon}(t, x)=E_{x} \varphi^{\epsilon}\left(t-\tau, \xi^{\epsilon}(\tau)\right) .
$$

When $\tau=t, \varphi_{\mu}{ }^{\epsilon}=\varphi^{\epsilon}=\Phi$. Thus

$$
\left|\varphi_{\mu}{ }^{\epsilon}(t, x)-\varphi^{\epsilon}(t, x)\right| \leqq 2\|\Phi\| P_{x}(\tau<t)
$$

for $x \in G$. By (4.7), if $x \in K, 0 \leqq t \leqq t_{1}$, then

$$
\left|\varphi_{\mu}^{\epsilon}(t, x)-\varphi^{\epsilon}(t, x)\right|=o\left(\epsilon^{k}\right)
$$

for any $k$. Since expansion (3.5) holds for $\varphi_{\mu}{ }^{\epsilon}$, it also holds for $\varphi^{\epsilon}$, uniformly on $\left[0, t_{1}\right] \times K$.

Step 3. It remains to remove the assumption that $\Phi$ is bounded. Choose $\Phi_{m}$ bounded for each $m=1,2, \cdots$, such that $\Phi_{m}=\Phi$ in $G_{m}$ and $\left|\Phi_{m}\right| \leqq|\Phi|$. Let

$$
\tilde{\varphi}_{m}{ }^{\epsilon}(t, x)=E_{x} \Phi_{m}\left[\xi^{\epsilon}(t)\right] .
$$

Then by (3.1a) and dominated convergence, $\tilde{\varphi}_{m}{ }^{\epsilon} \rightarrow \varphi^{\epsilon}$ as $m \rightarrow \infty$; also

$$
\left|\tilde{\varphi}_{m}{ }^{\epsilon}(t, x)\right| \leqq \mathrm{M}\left(1+E_{x} V\left[\xi^{\epsilon}(t)\right]^{\ell}\right) .
$$

By formula (A.2), the right side is bounded for $(t, x)$ in any compact set. A repetition of the reasoning in Step 2 shows that

$$
\left|\tilde{\varphi}_{\mu}{ }^{\epsilon}(t, x)-\varphi^{\epsilon}(t, x)\right| \leqq 2 M_{1} P_{x}(\tau<t),
$$

for suitable $M_{1}$. Consequently, by (4.7)

$$
\left|\tilde{\varphi}_{\mu} \epsilon^{\epsilon}(t, x)-\varphi^{\epsilon}(t, x)\right|=o\left(\epsilon^{k}\right)
$$


for any $k$. Since expansion (3.5) holds for $\tilde{\varphi}_{\mu}{ }^{\epsilon}$, it also holds for $\varphi^{\epsilon}$, uniformly on $\left[0, t_{1}\right] \times K$. This proves Theorem 4.2 .

5. Results for stopped processes. Let us consider now the autonomous form of $\left(4.1^{\epsilon}\right)$ :

$$
\mathcal{L}^{\epsilon} \varphi^{\epsilon}+F\left(x, \varphi^{\epsilon}\right)=0, \quad x \in B
$$

with boundary data

$$
\varphi^{\epsilon}(x)=\Phi(x), \quad x \in \partial B .
$$

Here $B$ is a given open set. For $\epsilon=0$, we get the first order equation

$$
\mathcal{L}^{0} \varphi^{0}+F\left(x, \varphi^{0}\right)=0 .
$$

The method of characteristics gives a $C^{\infty}$ solution to $\left(5.1^{0}\right)$ with the boundary data only for $x$ in certain portions of $B$ for which the trajectories $\xi^{0}$ exit from $B$ nontangentially. More precisely, let $\mathcal{O}$ be an open set, $N=\mathcal{O} \cap \bar{B}$, such that:

(i) $N \cap \partial B$ is a $C^{\infty}$ manifold;

(ii) if $x \in N$, then for the solution $\xi^{0}$ of $\left(2.1^{0}\right)$ with $\xi^{0}(0)=x$ we have $\xi^{0}(t) \in N$ for $0 \leqq t \leqq \tau^{0}$ and $\dot{\xi}^{0}\left(\tau^{0}\right)$ not tangent to $\partial B$ at $\xi^{0}\left(\tau^{0}\right)$.

The method of characteristics defines a $C^{\infty}$ solution $\varphi^{0}$ to $\left(5.1^{0}\right)$ in $N$, with $\varphi^{0}=\Phi$ on $N \cap \partial B$.

We suppose that $\varphi^{\epsilon}$ is a bounded solution of (5.1 $\left.)^{\epsilon}\right)-(5.2)$, of class $C^{2}$ in $B$ and continuous in the closure $\bar{B}$. We seek an expansion (3.5) valid in $N$, where the coefficients $\theta_{1}(x), \theta_{2}(x), \cdots$ satisfy in $N$ the first order partial differential equations obtained by putting $\left(\theta_{k}\right)_{t}=0$ in $\left(4.3^{0}\right)$. For boundary data, $\theta_{k}(x)=0$ on $N \cap \partial B$.

Formula (3.5) is the "regular expansion" in the theory of singular perturbations [4]

The proof of the following lemma is elementary. To emphasize the dependence on the initial data $x$, we write in this lemma $\tau^{0}=\tau^{0}(x)$.

LEMMA 5.1. Let $K$ be a compact subset of $N$, and $t_{1}>\tau^{0}(x)$ for all $x \in K$. Let $\tau=\tau(x)$ denote the minimum of $t_{1}$ and the exit time of $\xi^{\xi}$ from $N$. Then given $\eta>0$ there exists $a>0$ such that $\left\|\xi^{\epsilon}-\xi^{0}\right\|_{t_{1}}$ $\leqq$ a implies $\xi^{\epsilon}(\tau(x)) \in \partial B$ and $\left|\tau(x)-\tau^{0}(x)\right|<\eta$.

LemMA 5.2. If there exists $M$ such that $\left|\varphi^{\epsilon}(x)\right| \leqq M$ for all $x \in N$, then the expansion (3.5) holds uniformly on any compact subset of $N$.

To prove Lemma 5.2, only the following slight changes in the proof of Theorem 4.1 are needed. Define $\tau$ as in Lemma 5.1, and $\theta_{k}{ }^{\epsilon}$ as in $\$ 4$. We have as in $(4.8)$ 


$$
\theta_{k}^{\epsilon}(x)=E_{x} \int_{0}^{\tau} D^{\epsilon}(s)\left[\Gamma_{k}{ }^{\epsilon}+\operatorname{tr} \alpha\left(\theta_{k-1}\right)_{x x}\right] d x+E_{x}\left[D^{\epsilon}(\tau) \theta_{k}^{\epsilon}\left(\xi^{\epsilon}(\tau)\right)\right],
$$

where the integrand is evaluated at $\xi \epsilon(s)$. When $\xi \epsilon(\tau) \in \partial B$, $\theta_{k}{ }^{\epsilon}\left[\xi^{\epsilon}(\tau)\right]=0$; and $\left|\theta_{k}{ }^{\epsilon}\left[\xi^{\epsilon}(\tau)\right]\right| \leqq C_{k} \epsilon^{-k}$ when $\xi^{\epsilon}(\tau) \notin \partial B$. Let $a \leqq$ minimum distance to $B-N$ from any point $\xi^{0}(s), 0 \leqq s \leqq t_{1}$, on a trajectory $\xi^{0}$ starting from $x \in K$. Then

$$
P_{x}(\xi \epsilon(\tau) \notin \partial B) \leqq 2 n \exp \left(-a \epsilon^{-1 / 2} \lambda\right)
$$

for small $\epsilon$, by Lemma 2.1. Moreover, by Lemmas 2.1 and 5.1, $\left\|\xi^{\epsilon}-\xi^{0}\right\|_{t_{1}} \rightarrow 0$ and $\tau \rightarrow \tau^{0}$ in probability as $\epsilon \rightarrow 0$, uniformly for initial data $x \in K$. By the same reasoning as for Theorem 4.1, $\theta_{k}{ }^{\epsilon}(x) \rightarrow \theta_{k}(x)$ as $\epsilon \rightarrow 0$ uniformly on $K$. This proves Lemma 5.2.

For the homogeneous equation

$$
\mathcal{L}^{\epsilon} \varphi^{\epsilon}=0 \text { in } B
$$

with $\varphi^{\epsilon}=\Phi$ on $\partial B$, the maximum principle for elliptic equations implies that $\left\|\varphi^{\epsilon}\right\| \leqq\|\Phi\|$. Therefore, by Lemma 5.2:

Theorem 5.1. Let $\Phi$ be bounded, and let $N$ satisfy (5.3). Then the expansion (3.5) holds for bounded solutions of (5.5€)-(5.2), uniformly on each compact subset of $N$.

We note that, for some choices of $f$, Theorem 5.1 cannot be applied. At points $y \in N \cap \partial B$, we must have $f(y) \cdot \nu(y)>0$, where $\nu$ is the exterior unit normal to $\partial B$ at $y$. One possibility examined in [20], is when $f(y) \cdot \nu(y)<0$ for all $y \in \partial B$. In this case the drift term in $\left(2.1^{\epsilon}\right)$ impedes the movement of $\xi^{\epsilon}(t)$ towards $\partial B$. It is found in [20] that under certain assumptions $\varphi^{\epsilon}(x)$ tends to $\Phi\left(y^{*}\right)$ as $\epsilon \rightarrow 0$, where $y^{*}$ is a point of $\partial B$ making a certain functional on the unperturbed system trajectories minimum.

We next turn to the inhomogeneous equation. To obtain the result in this case an additional assumption about the mean exit time starting from any point in $B$ is needed.

THeOREM 5.2. Assume that: (1) F, $\Phi$ are bounded; (2) $N$ satisfies (5.3); and (3) $E_{x} \tau^{\epsilon} \leqq C \epsilon^{-1}$ for all $x \in B$, where $\tau^{\epsilon}$ is the exit time of $\xi \epsilon(t)$ from B. Then the expansion (3.5) holds uniformly on each compact subset of $N$.

Proof. Let $K$ be a compact subset of $N$. There exists $N_{1}$ with properties (5.3) such that $K \subset N_{1}$ and $\bar{N}_{1} \subset N$. We show that $\varphi^{\epsilon}$ is uniformly bounded on $N_{1}$. The theorem will then follow from Lemma 5.2 , with $N$ replaced by $N_{1}$ there. 
First of all, by Lemma 2.3

$$
\varphi^{\epsilon}(x)=E_{x} \int_{0}^{\tau^{\epsilon}} F\left[\xi^{\epsilon}(s), \varphi^{\epsilon}\left(\xi^{\epsilon}(s)\right)\right] d s+E_{x} \Phi\left[\xi^{\epsilon}\left(\tau^{\epsilon}\right)\right] .
$$

By assumptions (1) and (3) the crude estimate

$$
\left|\varphi^{\epsilon}(x)\right| \leqq C_{1} \epsilon^{-1}, \quad x \in B,
$$

holds for some $C_{1}$. For $x \in N_{1}$, we next let $\tau=\min \left(t_{1}\right.$, exit time of $\xi^{\epsilon}(t)$ from $\left.N\right)$, where $t_{1}>\tau^{0}(x)$ for all $x \in \bar{N}_{1}$. Again by Lemma 2.3,

$$
\varphi^{\epsilon}(x)=E_{x} \int_{0}^{\tau} F\left[\xi^{\epsilon}(s), \varphi^{\epsilon}\left(\xi^{\epsilon}(s)\right)\right] d s+E_{x}\left[\varphi^{\epsilon}\left(\xi^{\epsilon}(\tau)\right)\right] .
$$

For $\xi^{\epsilon}(\tau) \in \partial B, \varphi^{\epsilon}\left(\xi^{\epsilon}(\tau)\right)=\Phi\left(\xi^{\epsilon}(\tau)\right)$. Then

$$
\left|\varphi^{\epsilon}(x)\right| \leqq t_{1}\|F\|+\|\Phi\|+\left\|\varphi^{\epsilon}\right\| P_{x}\left(\xi^{\epsilon}(\tau) \notin \partial B\right) .
$$

Let $a \leqq$ distance $\left(N_{1}, B-N\right)$ be small enough that $\left\|\xi^{\epsilon}-\xi^{0}\right\|_{t_{1}} \leqq a$ implies $\xi^{\epsilon}(\tau) \in \partial B($ Lemma 5.1). By Lemma 2.1 and $(*)$

$$
\left\|\varphi^{\epsilon}\right\| P_{x}\left(\xi^{\epsilon}(\tau) \notin \partial B\right) \leqq C_{1} \epsilon^{-12 n} \exp \left(-a \epsilon^{-1 / 2} \lambda\right),
$$

which tends to 0 as $\epsilon \rightarrow 0$. Hence, $\left|\varphi^{\epsilon}(x)\right| \leqq M$ for all $x \in N_{1}$ and suitable constant $M$. This proves Theorem 5.2.

Remark. The proof shows that, instead of (3) we could equally well assume that

$$
E_{x} \tau^{\epsilon} \leqq C \epsilon^{-p} \text { for some } p>1 .
$$

It remains to discuss condition (3). Roughly speaking this condition is satisfied if the drift term $f$ in $\left(2.1^{\epsilon}\right)$ does not have too strong a tendency to keep $\xi \epsilon(s)$ inside $B$. For instance (3) cannot hold if there is some $B_{1} \subset B$ such that $f(y) \cdot \nu_{1}(y)<0$ for all $y \in \partial B_{1}$, where $\nu_{1}$ is the exterior unit normal to $\partial B_{1}$ at $y$. Suppose, however, that there is a function $h$ such that:

(i) In $B, h$ is $C^{2}$ and $h(x) \geqq 0$;

(ii) In $\bar{B}, h$ is continuous and bounded;

(iii) $\mathcal{L}^{\epsilon} h \leqq-c \epsilon$ in $B$, where $c>0$.

By Lemma 2.3,

$$
\begin{gathered}
h(x)=-E_{x} \int_{0}^{\tau^{\epsilon}} \mathcal{L}^{\epsilon} h\left[\xi^{\epsilon}(s)\right] d s+E_{x} h\left[\xi^{\epsilon}\left(\tau^{\epsilon}\right)\right], \\
h(x) \geqq c \epsilon E_{x} \tau^{\epsilon} .
\end{gathered}
$$


This gives the bound $E_{x} \tau^{\epsilon} \leqq c^{-1} \epsilon^{-1} h(x)$. Therefore, the existence of $h$ with properties (5.6) implies condition (3).

Example. Let $n=1, B=(a, b)$ a finite interval. Suppose $\alpha(x)>0$ on $[a, b], f(x)<0$ for $a \leqq x<\bar{x}, \quad f(x)>0$ for $\bar{x}<x \leqq b$. Choose $h$ with $h(a)=h(b)=0, h^{\prime \prime}<0, h^{\prime}(\bar{x})=0$. Since $f h^{\prime} \leqq 0$,

$$
\mathcal{L}^{\epsilon} h=\epsilon \alpha(x) h^{\prime \prime}+f(x) h^{\prime} \leqq \epsilon \alpha(x) h^{\prime \prime} \leqq-c \epsilon
$$

for some $c$. There is a regular expansion on $\left[a, x_{1}\right)$ and also one on $\left(x_{2}, b\right]$ if $x_{1}<\bar{x}<x_{2}$. By Theorem 5.2 with $F=1, E_{x} \tau^{\epsilon}$ is bounded except for $x$ near $\bar{x}$. However, if $x=\bar{x}$ (an unstable critical point for the unperturbed system $\left.\left(2.1^{0}\right)\right), E_{x} \tau^{\epsilon} \rightarrow \infty$ as $\epsilon \rightarrow 0$.

6. Population genetics models. In population genetics theory, several factors are considered as affecting the change in genetic makeup of a population with time. Among them are selective advantages held by individuals of one genotype over another, and rates of new mutations and of migration between habitats. Another factor is the random fluctuation in gene frequencies due to sampling at each generation. When the population size $N$ is so large that it can be taken as infinite, this factor is negligible; a deterministic model is then appropriate. However, for finite population size $\mathrm{N}$ a stochastic model is more appropriate.

The following model, taking into account finite population size has been studied considerably [2, Chaps. 8,9$]$. Consider one gene locus at which genes of either type $A_{1}$ or type $A_{2}$ may occur. In a diploid population (chromosomes in pairs) the three possible genotypes are $A_{1} A_{1}, A_{1} A_{2}, A_{2} A_{2}$. Let $x$ denote the frequency of gene $A_{1}$ initially, and $\xi(t)$ the frequency of gene $A_{1}$ in the $t$ th generation $(0 \leqq \xi(t) \leqq 1)$. The gene frequency process $\xi$ is modeled as a Markov chain, which is then approximated by a 1-dimensional diffusion process. The generator of this diffusion turns out to be of the form

$$
\mathcal{L} \phi=\frac{1}{4 N} x(1-x) \phi^{\prime \prime}+[x(1-x) \ell(x)+L(x)] \phi^{\prime},
$$

where $\ell(x)=c_{1} x+c_{2}, L(x)=c_{3} x+c_{4}$ are linear functions. (Actually in (6.1) instead of $N$ one should put the effective population size $N_{e}$.) The constants $c_{1}, c_{2}$ depend on the relative fitnesses of the three genotypes $A_{1} A_{1}, A_{1} A_{2}, A_{2} A_{2}$. When these are equally fit (no selective advantage), $\ell=0$. The constants $c_{3}, c_{4}$ depend on rates of new mutations of $A_{1}$ to $A_{2}$ or vice versa, or of migrations between the population in question and some outside pool of genes. When the effects of new 
mutations and migration are ignored, $L=0$. In the notation of the previous sections, $\mathcal{L}=\mathcal{L}^{\epsilon}, \xi=\xi \xi$, with

$$
\epsilon=\frac{1}{4 N}, \alpha(x)=x(1-x), f(x)=x(1-x) \ell(x)+L(x) .
$$

It is assumed that $N$ is constant (not dependent on $t$ ).

The probability distribution of $\xi(t)$ has been calculated in several cases, as an infinite series involving classical special functions [2, Chap. 8.4-8.6]. Instead of the probability distribution itself, one often wants expectations $E_{x} \Phi[\xi(t)]$ for various functions $\Phi$. In many instances these expectations can be found (exactly or approximately) more directly, with the aid of formula $\left(3.2^{\prime}\right)$. To illustrate this, we give two examples. In the second example we get at the same time, a known approximation to the smallest eigenvalue associated with the problem.

Example 1. Suppose $\ell(x)=0, f(x)=L(x)$. In population genetics literature, the selection pressures are then said to be linear. Let

$$
\begin{aligned}
\mu(t) & =E_{x} \xi(t) \\
H(t) & =E_{x} \xi(t)(1-\xi(t)) .
\end{aligned}
$$

Then $\mu(t)$ is the mean gene frequency at time $t$; the quantity $2 H(t)$ is called the heterozygosity at time $t$. From $\mu, H$ one also knows the variance $v(t)=\mu(t)-H(t)-\mu(t)^{2}$. By $\left(3.2^{\prime}\right)$ with $\Phi(x)=x$,

$$
\frac{d \mu}{d t}=E_{x} \mathcal{L} \Phi=E_{x} f
$$

Since $f=L, \mu$ satisfies the differential equation

$$
\frac{d \mu}{d t}=c_{3} \mu+c_{4}, \mu(0)=x .
$$

To get a differential equation for $H$, we take $\Phi(x)=x(1-x)$. Then

$$
\begin{aligned}
& \frac{d H}{d t}=E_{x} \mathcal{L} \Phi=E_{x}\left(f \Phi^{\prime}\right)+\epsilon E_{x}\left(\alpha \Phi^{\prime \prime}\right), \\
& f \Phi^{\prime}=c_{4}-\left(c_{3}+2 c_{4}\right) x+2 c_{3} x(1-x), \alpha \Phi^{\prime \prime}=-2 \Phi, \\
& \frac{d H}{d t}=c_{4}-\left(c_{3}+2 c_{4}\right) \mu+\left(2 c_{3}-2 \epsilon\right) H, H(0)=x(1-x) .
\end{aligned}
$$

In case $f=0$ (no selection pressure), this gives in particular the well known rate of decrease of heterozygosity $H(t)=H(0) \exp (-t / 2 N)$ since $2 \epsilon=(2 N)^{-1}$. See [2, p. 329] . 
Example 2. Suppose $L=0, f(x)=x(1-x) \ell(x)$. In this case new mutations and migrations are neglected. The nonlinearity of $f$ makes matters more difficult. Let us indicate a technique for calculating the mean heterozygosity approximately when $\ell \ll \epsilon$ (i.e., $\mathrm{Nl} \ll 1)$. For simplicity we carry out the calculations only when $c_{1}=0, \ell=$ constant. In genetical terms, this means that the fitness of the heterozygote $A_{1} A_{2}$ is the average of the fitness of the two homozygotes $A_{1} A_{1}$ and $A_{2} A_{2},[2$, p. 396]. Besides the function $H$ in Example 1, we introduce two other functions

$$
\begin{aligned}
H_{1}(t) & =E_{x}\left(f \Phi^{\prime}\right)=\ell E_{x}\left(\Phi \Phi^{\prime}\right), \\
H_{2}(t) & =E_{x}\left(f^{2}\right)=\ell^{2} E_{x} \Phi^{2}, \\
\Phi(x) & =\alpha(x)=x(1-x),
\end{aligned}
$$

where each expression inside an expectation $E_{x}$ is evaluated at $\xi(t)$. After calculating $\mathcal{L}\left(\boldsymbol{\Phi} \Phi^{\prime}\right), \mathcal{L} \Phi^{2}$, and using $\left(3.2^{\prime}\right)$ we get

$$
\begin{aligned}
\frac{d H}{d t} & =-2 \epsilon H+H_{1}, \\
\frac{d H_{1}}{d t} & =\ell^{2} H-6 \epsilon H_{1}-6 H_{2}, \\
\frac{d H_{2}}{d t} & =2 \epsilon \ell^{2} H-12 \epsilon H_{2}+2 \ell^{3} E_{x}\left(\Phi^{2} \Phi^{\prime}\right) .
\end{aligned}
$$

Since $\ell \ll \epsilon$, it can be seen that the term $2 \ell^{3} E\left(\Phi^{2} \Phi^{\prime}\right)$ can be neglected. One has then three linear differential equations for $H, H_{1}, H_{2}$. The solutions are linear combinations of exponentials $e^{\lambda_{i} t}$, where $\lambda_{1}, \lambda_{2}, \lambda_{3}$ are the characteristic roots of the coefficient matrix

$$
\left(\begin{array}{ccc}
-2 \epsilon & 1 & 0 \\
\ell^{2} & -6 \epsilon & -6 \\
2 \epsilon \ell^{2} & 0 & -12 \epsilon
\end{array}\right) .
$$

They satisfy the equation

$$
0=(\lambda+2 \epsilon)(\lambda+6 \epsilon)(\lambda+12 \epsilon)-\ell^{2} \lambda .
$$

If we denote by $\lambda_{1}$ the root of smallest absolute value, then with the aid of the implicit function theorem one finds that approximately for $\ell \ll \epsilon$

$$
\lambda_{1}=-\frac{1}{2 N}\left(1+\frac{2}{5}(N l)^{2}\right) .
$$


For $\left|\lambda_{1}\right| t$ large, the mean heterozygosity $2 H(t)$ decreases exponentially at rate nearly $\left|\lambda_{1}\right|$. Another interpretation for $\left|\lambda_{1}\right|$ is as the smallest eigenvalue of a boundary problem for the forward partial differential equation of the $\xi$ processes, stopped when the gene frequency $\xi(t)$ reaches either endpoint of the interval $[0,1]$. This follows from an asymptotic formula [2, p. 397] for the probability density of $\xi(t)$ when $\left|\lambda_{1}\right| t$ is large. Our formula for $\lambda_{1}$ agrees with the one in $[2, \mathrm{p}$. 398].

If $\ell$ is not constant, the same technique leads to five linear ordinary differential equations.

If $\epsilon \ll \ell$ (i.e., $N \ell \gg 1$ ), the mean heterozygosity in Example 2 could be estimated using the expansion (3.5) in powers of $\epsilon$. Times $t$ of order $\ell^{-1}$ are of interest in this case; for larger times the gene frequency $\xi(t)$ has reached 0 on 1 with probability very nearly 1 . (When $\epsilon \ll \ell$, the accuracy of the diffusion approximation to the genetical model is in doubt.)

Other questions about the gene frequency process $\xi$ can be answered by solving an ordinary differential equation

$$
\mathcal{L} \phi+g(x)=0, \quad 0<x<1,
$$

with given data for $\phi(0)$ and $\phi(1)$. Here $\mathcal{L}$ is the second order operator in (6.1). As in Example 2 we take $f=\ell x(1-x)$. If $g=0, \phi(0)=0$, $\phi(1)=1$, then $\phi(x)$ is the probability that gene $A_{2}$ becomes extinct eventually when $A_{1}$ initially has frequency $x$. If $g(x)=1, \phi(0)=\phi(1)$ $=0$, then $\phi(x)$ is the mean time until $A_{1}$ or $A_{2}$ becomes extinct. If $g(x)=x(1-x), \phi(0)=\phi(1)=0$, then

$$
\phi(x)=\int_{0}^{\infty} E_{x} \xi(t)(1-\xi(t)) d t
$$

and $2 N \phi(x)$ is the total mean number of heterozygotes.

An effective solution of the seemingly innocuous equation (6.3) is not generally easy. However, $\phi(x)$ is known at least approximately for various cases of interest [11, Chap. 1] [15]. We discuss here some cases when $\phi(x)$ can be found approximately from the expansion in $\S 5$, when $\epsilon=(4 N)^{-1}$ is small. Let us take as boundary data $\phi(0)=$ $\boldsymbol{\phi}(1)=0$. Then the probabilistic solution $\phi$ of $(6.3)$ is

$$
\phi(x)=E_{x} \int_{0}^{\tau} g[\xi(t)] d t,
$$

where $\tau$ is the time when $\xi(t)$ first reaches 0 or 1 . Let the relative fitness of the three genotypes $A_{1} A_{1}, A_{1} A_{2}, A_{2} A_{2}$ be respectively $1,1-h s$, 
$1-s$, where $s>0$. Then $\ell(x)=s[h+(1-2 h) x]$; in our previous notation, $c_{1}=s h, c_{2}=s(1-2 h)$.

Case 1 . $0 \leqq h \leqq 1$. Then $\ell(x)>0$ for $0<x \leqq 1$. If $g(1) \neq 0$, there is no regular expansion (3.5) of $\varphi\left(\approx \varphi^{\epsilon}\right)$ in powers of $\epsilon$. In fact, the first term in such an expansion could not be finite, since $0<\xi^{0}(t)<1$ for every $t$ and $\xi^{0}(t) \rightarrow 1$ as $t \rightarrow \infty$. Let us assume, therefore, that $g(1)=0$, and show that there is a regular expansion (3.5) on $[b, 1]$ for any $b>0$. Let us divide (6.2) by $1-x$, obtaining

$$
\begin{gathered}
\mathcal{L}^{*} \phi+g^{*}=0, \quad 0<x<1, \\
\mathcal{L}^{*} \phi=\epsilon x \phi^{\prime \prime}+x \ell(x) \phi^{\prime}, \quad g(x)=(1-x) g^{*}(x) .
\end{gathered}
$$

Consider, instead of $\xi$, a new diffision $\xi^{*}$ with generator $\mathcal{L}^{*}, f^{*}(x)=$ $x \ell(x), \alpha^{*}(x)=x$. The assumptions (5.3) are satisfied by $f^{*}$ on $(b, 1]$. By the Remark after Theorem 5.2 there is a regular expansion on $(b, 1]$ provided $E_{x} \tau^{*} \leqq C \epsilon^{-2}$ for some $C$, and for $0<x<1$. Here $\tau^{*}$ is the exit time of $\xi^{*}(t)$ from $(0,1)$. Let

$$
\psi(x)=\epsilon^{2} E_{x} \tau^{*}, \quad m(x)=\epsilon^{-1} \int_{0}^{x} \ell(y) d y .
$$

Since $\ell>0, m$ is increasing. Moreover,

$$
\psi^{\prime \prime}+m^{\prime} \psi^{\prime}+\frac{\epsilon}{x}=0 .
$$

The following function $\psi$ is also a solution of (\#):

$$
\tilde{\psi}(x)=-\epsilon \int_{0}^{x} e^{-m(y)}\left[\int_{0}^{y} \frac{e^{m(z)}-1}{z} d z+\log y\right] d y .
$$

By the mean value theorem, monotonicity of $m$, and $m^{\prime}=\epsilon^{-1} \ell$, we have

$$
\frac{e^{m(z)}-1}{z} \leqq \epsilon^{-1}\|\ell\| e^{m(z)} .
$$

It follows that $|\tilde{\psi}(x)| \leqq(1 / 2) C$, for some $C$ not depending on $\epsilon$. Then $(1 / 2) C+\tilde{\psi}$ is a nonnegative solution of $(\#)$. Since $\psi$ is also a solution of $(\#)$, with $\psi(0)=\psi(1)=0$, we have $\psi \leqq(1 / 2) C+\tilde{\psi} \leqq C$. Thus $E_{x} \tau^{*} \leqq C \epsilon^{-2}$ as required.

Case 2. $h>1$. Assume that $g(0)=g(1)=0$, and divide (6.2) by $x(1-x)$. Then

$$
\begin{gathered}
\mathcal{L}^{* *} \phi+g^{* *}=0, \quad 0<x<1, \\
\mathcal{L}^{* *} \phi=\epsilon \phi^{\prime \prime}+\ell(x) \phi^{\prime}, \quad g(x)=x(1-x) g^{* *}(x) .
\end{gathered}
$$


This is the situation in the Example at the end of $\$ 5$, if we take $a=0$, $b=1$ there $\alpha^{* *}(x)=1, f^{* *}(x)=\ell(x), \bar{x}=h(2 h-1)^{-1}$. In this case, there is a regular expansion about each of the endpoints 0,1 .

Case 3. $h<0$. The heterozygote $A_{1} A_{2}$ is more fit than either homozygote (overdominance). The term $f d t$ in $\left(2.1^{\epsilon}\right)$ has a strong tendency to drive $\xi(t)$ toward $\bar{x}$, which is a stable equilibrium point when $\epsilon=0$ (in Case 2, $\bar{x}$ is unstable). The mean exit time $E_{x} \tau^{\epsilon}$, for $x$ outside a neighborhood of the endpoints 0,1 , is at least of order $\exp \left(c \epsilon^{-1}\right)$ for some $c>0$. There is no regular expansion. On the contrary, from (6.4) one can see that $\phi(x)$ is also extremely large if $g(x)>0$ for $0<x<1$.

Multidimensional genetic models. The model considered above dealt with two kinds of gene $A_{1}$ or $A_{2}$ (called alleles) at a single locus. The resulting diffusion is 1-dimensional, if the population size $N$ is assumed fixed. Models leading to multidimensional diffusions arise in several ways. For instance, the diffusion approximation will be multidimensional in case of several alleles at a single locus, or if more than one gene locus is considered, or if different gene frequencies at different habitats are taken into account.

If $\mathcal{L}$ denotes the generator of an $n$-dimensional diffusion approximation in one of these models, frequently the second order coefficients $\alpha_{i j}(x)$ are quadratic in $x=\left(x_{1}, \cdots, x_{n}\right)$. If, moreover, $f$ is linear, then the means and covariances of $\xi_{1}(t), \cdots, \xi_{n}(t)$ satisfy first order differential equations. These equations are found in a way similar to that for Example 1, $\S 3$ and Example 1, $\S 6$. There are also interesting results for 2 locus models. In particular, the mean and variance of the linkage disquilibrium have been computed, by methods in the same spirit as for Example 2, $\$ 6$. See [11, Chap. 7]. However, it seems that much remains to be done in the study of multidimensional genetic models.

\section{APPENDIX}

We outline here proofs of some statements made in $\S 2$. It is always assumed that $0 \leqq \epsilon \leqq 1$. For notational simplicity, we write in the Appendix $\xi^{\epsilon}=\xi, \mathcal{L}^{\epsilon}=\mathcal{L}$, not showing their dependence on $\epsilon$.

First of all, given any smooth coefficients $f, \sigma$, a solution of $\left(2.1^{\epsilon}\right)$ can be constructed in the following way, up to some "explosion time" $\tau^{\infty}$. Let $G_{1} \subset G_{2} \subset \cdots$ be open bounded sets with union $R^{n}$, and $f_{m}, \sigma_{m}$ satisfying the Ito conditions for $m=1,2, \cdots$ such that

$$
f_{m}(x)=f(x), \sigma_{m}(x)=\sigma(x), \quad x \in G_{m} .
$$

For these coefficients there is a solution process $\xi_{m}$. Moreover, if $x \in G_{\mu}$ with $\mu<m$, then with probability $1, \xi_{\mu}(t)=\xi_{m}(t)$ for $0 \leqq t \leqq$ 
$\tau_{\mu}$ where $\tau_{\mu}$ is the exit time from $G_{\mu}$. See [3, Chap. 11, $\left.\S 3\right]$. Define $\tau^{\infty}$ as the limit of the monotone sequence $\tau_{m}$, and $\xi(t)=\xi_{m}(t)$ for large enough $m$ and $0 \leqq t<\tau^{\infty}$. It is known that assumptions $(2.4 \mathrm{a})(2.4 \mathrm{c})$ guarantee that $\tau^{\infty}=\infty$, and in fact that $E_{x} V[\xi(t)]<\infty$ for any $t \geqq 0$. See [13, Chap. II.2, Chap. III]. For completeness, we include the following proof. Take

$$
f_{m}=f \beta_{m}{ }^{2}, \sigma_{m}=\sigma \beta_{m}, G_{m}=\{x: V(x)<m\},
$$

where $0 \leqq \beta_{m} \leqq 1, \beta_{m}(x)=1$ for $x \in G_{m}, \beta_{m}(x)=0$ for $x \notin G_{m+1}$. The generator $\mathcal{L}_{m}$ of the process $\xi_{m}$ satisfies $\mathcal{L}_{m}=\beta_{m}{ }^{2} \mathcal{L}$, and hence $\mathcal{L}_{m} V \leqq M(1+V)$ with the same constant $M$ in (2.4a). Let

$$
\rho_{m}(t)=E_{x} V\left[\xi_{m}(t)\right] .
$$

From the Ito stochastic differential rule we have (as in formula 3.2)

$$
\rho_{m}(t)=\rho_{m}(0)+\int_{0}^{t} E_{x} \mathcal{L}_{m} V\left[\xi_{m}(s)\right] d s,
$$

and therefore by $(2.4 \mathrm{a})$

$$
\rho_{m}(t) \leqq \rho_{m}(0)+M \int_{0}^{t}\left(1+\rho_{m}(s)\right) d s .
$$

Since $\rho_{m}(0)=V(x)$, Gronwall's inequality gives the bound independent of $m$ :

$$
\rho_{m}(t) \leqq(V(x)+M t) e^{M t}
$$

Let

$$
W(t, x)=e^{M t} V(x)+e^{M t}-1 .
$$

Then $-W_{t}+\mathcal{L}_{m} W \leqq 0$, which implies that $W\left(t-s, \xi_{m}(s)\right)$ is a nonnegative supermartingale. Therefore,

$$
P_{x}\left(\max _{0 \leqq s \leqq t} W\left(t-s, \xi_{m}(s)\right) \geqq m\right) \leqq \frac{1}{m} E_{x} W\left(0, \xi_{m}(t)\right) .
$$

The right side is $m^{-1} \rho_{m}(t)$. Since $V \leqq W$ and $\tau_{m}$ is the exit time from $\{V<m\}$,

$$
P_{x}\left(\tau_{m}>t\right) \leqq m^{-1} \rho_{m}(t) .
$$

By $(*), P_{x}\left(\tau_{m}>t\right) \rightarrow 0$ as $m \rightarrow \infty$. Hence $\tau^{\infty}=\infty$. Since $V\left[\xi_{m}(t)\right]$ $=V[\xi(t)]$ for large enough $m$ (with probability 1), Fatou's lemma together with $(*)$ imply

$$
E_{x} V[\xi(t)] \leqq(V(x)+M t) e^{M t} .
$$


Let us next show, for any positive integer $\ell$, a similar estimate

$$
E_{x} V^{\ell}[\xi(t)] \leqq\left(V^{\ell}(x)+M_{\ell} t\right) e^{M_{\ell} t} .
$$

For this it suffices to show that $V^{\ell}$ also satisfies (2.4a) with some constant $M_{\ell}$. By direct calculation,

$$
\mathcal{L} V^{\ell}=\ell V^{\ell-1} \mathcal{L} V+\ell(\ell-1) V^{\ell-2} V_{x} \alpha V_{x} .
$$

By $(2.3),|\alpha(x)| \leqq M^{2}(1+|x|)^{2}$; while by $(2.4 \mathrm{~b})$

$$
\begin{aligned}
(1+|x|)^{2}\left|V_{x}\right|^{2} & \leqq M^{2}(1+V)^{2}, \\
V^{\ell-2} V_{x} \alpha V_{x} & \leqq V^{\ell-2}\left|V_{x}\right|^{2}|\alpha|,
\end{aligned}
$$

which gives with suitable $M_{\ell}$

$$
\mathcal{L} V^{\ell} \leqq M_{\ell}\left(1+V^{\ell}\right)
$$

as required.

Proof of Lemma 2.1. Given any compact set $K \subset R^{n}$ and $t_{1}>0$ let $K_{1}$ be the compact set consisting of all points $\xi^{0}(s), 0 \leqq s \leqq t_{1}$, on the solution $\xi^{0}$ of $\left(2.1^{\circ}\right)$ starting from some $x \in K$. Given $a>0$ and $0 \leqq$ $t \leqq t_{1}, P_{x}\left(\left\|\xi^{\epsilon}-\xi^{0}\right\|_{t}>a\right)$ is unaffected if we change $f, \sigma$ outside the $a$-neighborhood of $K_{1}$. Hence we may assume that $f, \sigma$ are bounded and Lipschitz on $R^{n}$.

Let us write $\left(2.1^{\mathrm{\epsilon}}\right),\left(2.1^{0}\right)$ in integrated form with the initial data (2.2) and subtract them. We get

$$
\begin{aligned}
\xi^{\epsilon}(t)-\xi^{0}(t) & =\int_{0}^{t}\left[f\left(\xi^{\epsilon}(s)\right)-f\left(\xi^{0}(s)\right)\right] d s+\epsilon^{1 / 2} \zeta(t), \\
\zeta(t) & =2^{1 / 2} \int_{0}^{t} \sigma\left(\xi^{\epsilon}(s)\right) d w .
\end{aligned}
$$

By standard estimates,

$$
\left\|\xi^{\epsilon}-\xi^{0}\right\|_{t} \leqq \epsilon^{1 / 2}\|\zeta\|_{t} e^{k t}
$$

where $k$ is a Lipschitz constant for $f$. Thus,

$$
P_{x}\left(\left\|\xi^{\epsilon}-\xi^{0}\right\|_{t}>a\right) \leqq P_{x}\left(\epsilon^{1 / 2}\|\zeta\|_{t} e^{k t}>a\right) .
$$

To estimate the right side, we use an exponential martingale inequality. For $\gamma \in R^{n}$ with $|\gamma|=1$, let

$$
\chi(t)=\gamma \cdot \zeta(t)-\int_{0}^{t}|\gamma \sigma|^{2} d s .
$$

Then $[16$, p. 25] 


$$
P_{x}\left(\max _{0 \leqq s \leqq t} \chi(s)>c\right) \leqq e^{-c} .
$$

Since $\sigma$ is bounded, $|\gamma \sigma|^{2} \leqq B$ for some $B$. Hence $\gamma \zeta(s)>c+B t$ implies $\chi(s)>c$. Take $\gamma= \pm e_{i}$, where $e_{1}, \cdots, e_{n}$ is an orthonormal basis for $R^{n}$. Then $\|\zeta\|_{t}>d$ implies $\max _{0 \leqq s \leqq t} \gamma \zeta(s)>n^{-1 / 2} d$ for at least one of these $2 n$ choices for $\gamma$. By taking $d=n^{1 / 2}(c+B t)$, we get

$$
\begin{aligned}
P_{x}\left(\|\zeta\|_{t}>n^{1 / 2}(c+B t)\right) \leqq & \sum_{i=1}^{n}\left[P_{x}\left(\max \zeta_{i}(s)>c+B t\right)\right. \\
& \left.+P_{x}\left(\min \zeta_{i}(s)<-c-B t\right)\right] \leqq 2 n e^{-c}
\end{aligned}
$$

Take

$$
c=a \epsilon^{-1 / 2} \lambda, \lambda=\frac{1}{2} n^{-1 / 2} e^{-k t_{1}} .
$$

For small enough $\epsilon$,

$$
\begin{aligned}
n^{1 / 2}(c+B t) & \leqq a \epsilon^{-1 / 2} e^{-k t}, \quad 0 \leqq t \leqq t_{1}, \\
P_{x}\left(\left\|\xi^{\epsilon}-\xi^{0}\right\|_{t}>a\right) & \leqq P_{x}\left(\|\zeta\|_{t}>n^{1 / 2}(c+B t) \leqq 2 n \exp \left(-a \epsilon^{-1 / 2} \lambda\right) .\right.
\end{aligned}
$$

This proves Lemma 2.1.

Proof of Lemma 2.2. Step 1. Suppose that $A, \psi_{t}, \psi_{x_{i}}, \psi_{x_{i} x_{j}}$ are continuous and bounded everywhere. Then

$$
d(D \psi)=(d D) \psi+D d \psi,
$$

where $\psi$ is evaluated at $(t-s, \xi(s))$ for fixed $t$. The ordinary product rule holds since the paths $D(\cdot)$ are $C^{1}$ functions of time. Now

$$
\begin{aligned}
d D & =A D d s, \\
d \psi & =\left(-\psi_{t}+\mathcal{L} \psi\right) d s+(2 \boldsymbol{\epsilon})^{1 / 2} \psi_{x} \sigma d w, \\
d(D \psi) & =D\left[-\psi_{t}+\mathcal{L} \psi+A \psi\right] d s+D(2 \boldsymbol{\epsilon})^{1 / 2} \psi_{x} \sigma d w .
\end{aligned}
$$

We take $E_{x} \int_{0}^{\tau}$ of both sides, and use $D(0)=1, \xi(0)=x$, and (2.5). Since $D \psi_{x} \sigma$ is bounded and $\tau$ nonanticipative

$$
E_{x} \int_{0}^{\tau} D \psi_{x} \sigma d w=0
$$

This gives (2.6) under our present assumptions on $A, \psi_{t}, \cdots$.

Step 2. To obtain (2.6) under the assumptions in Lemma (2.2), consider open sets $G_{1}{ }^{\prime}, G_{2}{ }^{\prime}, \cdots$ with union $G, \bar{G}_{m}{ }^{\prime} \subset G_{m+1}{ }^{\prime}$, and $\bar{G}_{m}{ }^{\prime}$ compact. There exist $A_{m}, \psi_{m}$ satisfying the assumptions in Step 1 and agreeing on $G_{m}{ }^{\prime}$ with $A, \psi$. Then (2.6) holds with $\tau$ replaced by 
$\tau_{m}{ }^{\prime}=\min \left(t\right.$, exit time of $\xi(s)$ from $\left.G_{m}{ }^{\prime}\right)$. Moreover, $\tau_{m}{ }^{\prime}$ increases to $\tau$ as $m \rightarrow \infty$. We get (2.6) from the dominated convergence theorem. This proves Lemma 2.2 .

\section{REFERENCES}

1. G. Carrier, Stochastically perturbed dynamical systems, J. Fluid Mechanics 44 (1970), 249-264.

2. J. Crow and M. Kimura, Introduction to Population Genetics Theory, Harper and Row, 1970.

3. E. B. Dynkin, Markov Processes, Springer-Verlag, 1965.

4. W. Eckhaus, Boundary layers in linear elliptic singular perturbation problems, SIAM Review 14 (1972), 225-270.

5. W. H. Fleming, Optimal continuous-parameter stochastic control, SIAM Review 11(1969), 470-509.

6. - Stochastic control for small noise intensities, SIAM J. Control 9 (1971), 473-517.

7. _ Dynamical systems with small stochastic terms, Techniques of Optimization, Academic Press, 1972, pp. 325-333.

8. A. Friedman, Partial Differential Equations of Parabolic Type, PrenticeHall, 1964

9. I. Gikhman and A. Skorokhod, Introduction to the Theory of Random Processes, Saunders, 1969.

10. — Stochastic Differential Equations, Springer Verlag, 1972.

11. M. Kimura and T. Ohta, Theoretical Aspects of Population Genetics, Princeton University Press, 1971.

12. A. Kolmogorov, I. Petrovsky, and N. Piscounoff, Étude de l'équation de la diffusion avec croissance de la quantité de matière et son application a un probleme biologique, Bull. Univ. d'Etat Moscou Ser. Internat. Sec. A, 1 (1937), 1-26.

13. H. Kushner, Stochastic Stability and Control, Academic Press, New York, 1967.

14. - Stability and existence of diffusions with discontinuous or rapidly growing drift terms, J. Diff. Eq. 11 (1972), 156-168.

15. W. H. Li and M. Nei, Total number of individuals affected by the single deleterious mutation in a finite population, American J. Human Genetics 24 (1972), 667-679.

16. H. P. McKean, Jr., Stochastic Integrals, Academic Press, 1969.

17. G. C. Papanicolaou, Some problems and methods for the analysis of stochastic equations, Proceedings of Amer. Math. Soc. Symposium on Stochastic Differential Equations in New York, March 1972.

18. G. C. Papanicolaou and J. B. Keller, Stochastic differential equations with applications to random harmonic oscillators, SIAM J. Appl. Math. 21 (1971), 287305.

19. R. L. Stratonovich, Topics in the Theory of Random Noise, Vol. I, II, Gordon and Breach, New York, 1963.

20. A. D. Ventsel and M. I. Freidlin, On diffusion motion of a particle against a flow, Uspekhi Mat. Nauk NS 24, No. 5 (1969), 229-230.

Brown University, Providence, R.I. 02912 
